\title{
How Amphipols Embed Membrane Proteins: Global Solvent Accessibility and Interaction with a Flexible Protein Terminus
}

\author{
Manuel Etzkorn • Manuela Zoonens • \\ Laurent J. Catoire $\cdot$ Jean-Luc Popot • \\ Sebastian Hiller
}

Received: 24 January 2014/ Accepted: 7 March 2014/Published online: 26 March 2014

(c) Springer Science+Business Media New York 2014

\begin{abstract}
Amphipathic polymers called amphipols provide a valuable alternative to detergents for keeping integral membrane proteins soluble in aqueous buffers. Here, we characterize spatial contacts of amphipol A8-35 with membrane proteins from two architectural classes: The 8 -stranded $\beta$-barrel outer membrane protein OmpX and the $\alpha$-helical protein bacteriorhodopsin. OmpX is well structured in A8-35, with its barrel adopting a fold closely similar to that in dihexanoylphosphocholine micelles. The accessibility of A8-35-trapped OmpX by a water-soluble paramagnetic molecule is highly similar to that in detergent micelles and resembles the accessibility in the natural membrane. For the $\alpha$-helical protein bacteriorhodopsin, previously shown to keep its fold and function in amphipols, NMR data show that the imidazole protons of a polyhistidine tag at the N-terminus of the protein are exchange protected in the presence of detergent and lipid bilayer nanodiscs, but not in amphipols, indicating the absence of an interaction in the latter case. Overall, A8-35 exhibits protein interaction properties somewhat different from detergents and lipid bilayer nanodiscs, while maintaining the structure of solubilized integral membrane proteins.
\end{abstract}

\section{Etzkorn}

Institute of Physical Biology, Heinrich Heine University,

Universitätsstr. 1, 40225 Düsseldorf, Germany

M. Zoonens - L. J. Catoire - J.-L. Popot

UMR 7099, Institut de BiologiePhysico-Chimique,

CNRS/Université Paris-7, FRC 550, 13 rue Pierre et Marie

Curie, 75005 Paris, France

S. Hiller $(\bowtie)$

Biozentrum, University of Basel, Klingelbergstr. 70,

4056 Basel, Switzerland

e-mail: sebastian.hiller@unibas.ch
Keywords NMR spectroscopy · Membrane proteins . Paramagnetic surface mapping · OmpX .

Bacteriorhodopsin

\begin{tabular}{|c|c|}
\hline \multicolumn{2}{|l|}{ Abbreviations } \\
\hline A8-35 & $\begin{array}{l}\text { Polyacrylate-based amphipol, carrying } \\
\sim 25 \% \text { octylamide chains, } \\
\sim 40 \% \text { isopropyl-amide ones, } \\
\text { and } \sim 35 \% \text { free carboxylates }\end{array}$ \\
\hline APol & Amphipol \\
\hline BR & Bacteriorhodopsin \\
\hline DDM & $n$-Dodecyl- $\beta$-D-maltopyranoside \\
\hline DHPC & $\begin{array}{l}\text { 1,2-Dihexanoyl-sn-glycero-3- } \\
\text { phosphocholine }\end{array}$ \\
\hline DMPC & $\begin{array}{l}\text { 1,2-Dimyristoyl-sn-glycero-3- } \\
\text { phosphocholine }\end{array}$ \\
\hline $\begin{array}{l}\mathrm{Gd}(\text { DOTA })^{-}= \\
\text {DOTAREM }\end{array}$ & $\begin{array}{l}\text { A gadolinium ion chelated with } 1,4, \\
7,10 \text {-tetraazocyclododecane- } N, N^{\prime}, N^{\prime \prime}, \\
N^{\prime \prime \prime} \text {-tetraacetic acid }\end{array}$ \\
\hline OmpX & Outer membrane protein $\mathrm{X}$ \\
\hline
\end{tabular}

\section{Introduction}

Biophysical and biochemical studies of integral membrane proteins are of key interest for characterizing their structure and function. Because membrane proteins are adapted to a hydrophobic phase, keeping them soluble in aqueous solutions is an intrinsic challenge, requiring the use of surfactants that can substitute the hydrophobic environment of a natural membrane (Raschle et al. 2010). Whereas detergent micelles represent the classical case, they can be destabilizing to some membrane proteins or to protein- 
protein interactions. A number of alternative, milder systems have therefore been developed, such as lipid bilayer nanodiscs or amphipols (APols) (Bayburt et al. 2002; Bayburt and Sligar 2010; Popot et al. 2011; Tribet et al. 1996; Zoonens and Popot 2014). Results from multiple techniques, including NMR spectroscopy (Catoire et al. 2010, 2009; Planchard et al. 2014; Zoonens et al. 2005), electron microscopy (Althoff et al. 2011; Huynh et al. 2014; Liao et al. 2013) and molecular dynamics simulation (Perlmutter et al. 2014) indicate that APols associate with membrane proteins and keep them water soluble by adsorbing specifically onto their hydrophobic transmembrane surface. Structure, dynamics and function of a membrane protein may, however, depend on the surfactant type (Champeil et al. 2000; Dahmane et al. 2013; Elter et al. 2014; Etzkorn et al. 2013; Martinez et al. 2002; Perlmutter et al. 2014; Popot et al. 2011).

Here, we use high-resolution NMR spectroscopy to characterize the interactions of A8-35, a polyacrylate-based APol (Tribet et al. 1996) with proteins from two architectural classes, the $\beta$-barrel Escherichia coli outer membrane protein $\mathrm{X}(\mathrm{OmpX})$ and the $\alpha$-helical bacteriorhodopsin (BR) from Halobacterium salinarum. Whereas previous NMR characterizations of OmpX/APol contacts were based on amide proton protection factors (Catoire et al. 2010), we are using here paramagnetic surface mapping, which provides a complementary and more extensive coverage. In addition, we examine the interaction of a polyhistidine (poly-His) tag with three different surfactants, APols, lipid bilayer nanodiscs and detergent micelles. Poly-His tags are of widespread use for protein purification and thus are frequently genetically fused to proteins. Because they are flexible, unstructured and relatively polar, they are expected to remain water accessible. However, in the case of membrane proteins, the uncharged nature of poly-His tags at alkaline $\mathrm{pH}$ can give rise to an interaction with the surfactants associated with the protein. We investigate, here, whether APol A8-35 interacts with a poly-His tag fused to the N-terminus of BR.

\section{Materials and Methods}

$\left[U_{-}{ }^{15} \mathrm{~N}, \sim 75 \%-{ }^{2} \mathrm{H}\right]$-labeled OmpX was produced by overexpression in E. coli BL21(DE3)pLysS cells transformed with the plasmid pET3b-OmpX (Vogt and Schulz 1999). Cells were grown in $\mathrm{D}_{2} \mathrm{O}$-based $\mathrm{M} 9$ minimal medium containing ${ }^{15} \mathrm{NH}_{4} \mathrm{Cl}$ as the sole nitrogen source. Cells were induced after reaching an $\mathrm{OD}_{600}$ of 0.8 and harvested after $4 \mathrm{~h}$ by centrifugation. OmpX was purified from inclusion bodies and refolded into DHPC (1,2-dihexanoyl-sn-glycero-3-phosphocholine) micelles as described (Fernández et al. 2004). Partially, deuterated A8-35 (batch DAPol-4) was synthesized by F. Giusti (UMR 7099) following published protocols
(Gohon et al. 2006, 2004) and added to the protein solution. Subsequently, the detergent was removed using BioBeads, following standard protocols (Zoonens et al. 2005). The remaining amount of detergent in the sample was assessed by $1 \mathrm{D}^{1} \mathrm{H}$ NMR measurements. The final NMR sample contained $1 \mathrm{mM}$ OmpX with deuterated A8-35 in a standard NMR buffer (20 mM sodium phosphate, $100 \mathrm{mM} \mathrm{NaCl}, 5 / 95 \%$ $\mathrm{D}_{2} \mathrm{O} / \mathrm{H}_{2} \mathrm{O}, \mathrm{pH}$ 8.0). Titration of the OmpX solution was performed by adding the paramagnetic relaxation enhancement agent $\mathrm{Gd}(\mathrm{DOTA})^{-}$(DOTAREM; a gadolinium ion chelated with 1,4,7,10-tetraazocyclododecane- $N, N^{\prime}, N^{\prime \prime}, N^{\prime \prime \prime}$-tetraacetic acid; Laboratoire Guerbet, France) from an aqueous stock solution. The 2D $\left[{ }^{15} \mathrm{~N},{ }^{1} \mathrm{H}\right]-\mathrm{TROSY}$ spectra for the Gd(DOTA $)^{-}$titration were recorded with 16 transients per increment, $t_{1 \max }\left({ }^{15} \mathrm{~N}\right)=44 \mathrm{~ms}, t_{2 \max }\left({ }^{1} \mathrm{H}\right)=96 \mathrm{~ms}$, at $30{ }^{\circ} \mathrm{C}$ on a Bruker DRX $750 \mathrm{MHz}$ NMR spectrometer with a triple resonance TXI probe. Measurement of the rotational correlation time was taken with the TRACT method (Lee et al. 2006). BR was expressed using a cell-free expression system and was refolded into DDM detergent micelles, DMPC lipid bilayer nanodiscs or A8-35 following published protocols (Etzkorn et al. 2013). NMR spectra of BR in the different surfactant environments were recorded under identical buffer conditions (20 mM sodium phosphate $\mathrm{pH} 7.3,50 \mathrm{mM} \mathrm{NaCl}$, $\left.8 \% \mathrm{D}_{2} \mathrm{O}\right)$.

\section{Results and Discussion}

The 8 -stranded $\beta$-barrel membrane protein OmpX was refolded into dihexanoylphosphocholine (DHPC) detergent micelles (Fernández et al. 2004; Pautsch et al. 1999) and subsequently transferred to A8-35 (Catoire et al. 2009). The correct fold of the protein in A8-35 was assessed by comparing the $2 \mathrm{D}\left[{ }^{15} \mathrm{~N},{ }^{1} \mathrm{H}\right]-\mathrm{TROSY}$ spectra of OmpX/A835 and OmpX/DHPC (Fig. 1a, b). The glycine resonances, which are upfield in the nitrogen dimension, as well as the $\beta$-sheet peaks, which are shifted downfield in the proton dimension, were found to feature highly similar chemical shifts in both spectra and thus virtually identical peak patterns resulted for 71 amide moieties. The high similarity of resonance frequencies for large parts of the protein backbone allows unambiguous transfer of resonance assignments from OmpX in DHPC micelles to OmpX/A835 complexes and, at the same time, directly indicates identical secondary and tertiary structure for these residues under both conditions. Importantly, most of the identified residues are located in the $\beta$-barrel region, confirming that the protein adopts the same fold in both environments.

Measurements of the ${ }^{1} \mathrm{H}$ amide proton resonance line widths show that these are increased in the complex with A8-35 amphipols compared with DHPC by a factor of approximately 2.0 (Fig. 1). Broader resonance lines of 
Fig. 1 Global fold

characterization of OmpX in partially deuterated A8-35. 2D

$\left[{ }^{15} \mathrm{~N},{ }^{1} \mathrm{H}\right]$-TROSY spectra of a $1 \mathrm{mM}\left[U_{-}{ }^{15} \mathrm{~N}, 75 \%{ }^{2} \mathrm{H}\right]-$ OmpX in complex with deuterated A8-35 amphipol and b OmpX in DHPC solution, both recorded at $30{ }^{\circ} \mathrm{C}$. c $1 \mathrm{D}$ cross sections of two glycine resonances marked with an arrow in panels $\mathbf{a}$ and

b. d TRACT analysis. Symbols represent for experimental data (integrals of the region 8.5-10.5 ppm, circles $=R_{\alpha}$, squares $=R_{\beta}$ ). Black lines represent exponential fits, resulting in $R_{\alpha}=33 \mathrm{~Hz}$, $R_{\beta}=129 \mathrm{~Hz}$ and $\tau_{\mathrm{c}}=39 \mathrm{~ns}$
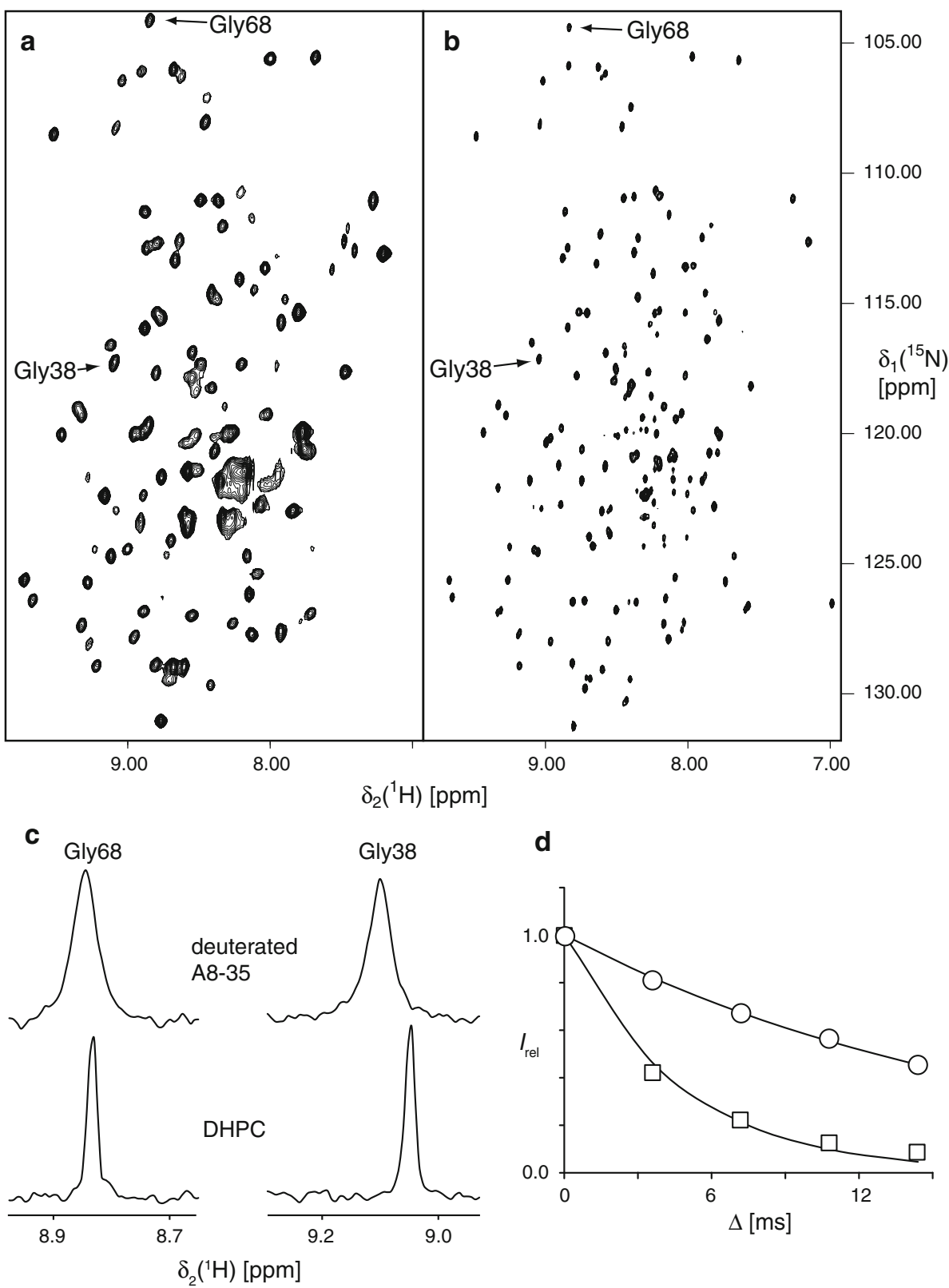

d

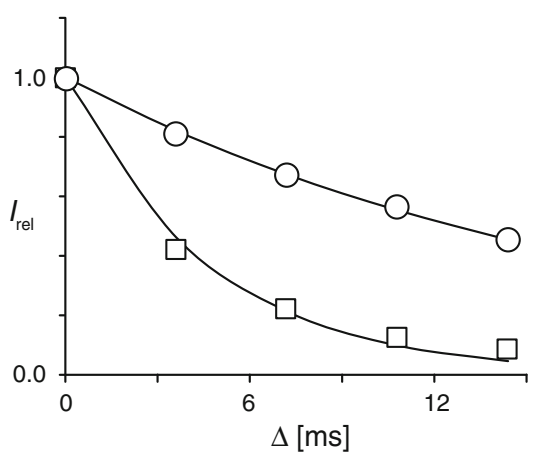

$\beta$-barrel membrane proteins in A8-35 as compared with detergent micelles have previously been observed for OmpX (Catoire et al. 2010) and for the transmembrane domain of OmpA (Zoonens et al. 2005). We address the contributions of decreased Brownian motion to the line width by measurements of the rotational correlation time $\tau_{\mathrm{c}}$ with the TRACT method (Lee et al. 2006). Thereby, $\tau_{\mathrm{c}}$ was found to be $39 \pm 5 \mathrm{~ns}$ in our OmpX/A8-35 preparations, which are larger by a factor of 1.85 than the value of $21 \mathrm{~ns}$ reported in DHPC micelles (Lee et al. 2006). Since the resonance line width scales in first order linearly with $\tau_{\mathrm{c}}$, these measurements can largely rationalize the increased ${ }^{1} \mathrm{H}$ line width. Interestingly, previously reported preparations of OmpX/A8-35 complexes featured a $\tau_{\mathrm{c}}$ value of
31 ns (Catoire et al. 2010). This difference may be attributed to the absence of EDTA in our sample buffer. EDTA has been shown to sequester residual $\mathrm{Ca}^{2+}$ ions, which can bridge A8-35 particles involved in complexes with membrane proteins, reducing molecular tumbling (Picard et al. 2006; Catoire et al. 2010). For solution NMR studies, it should thus be generally recommended to include EDTA in the preparations of MP/A8-35 samples.

The surface accessibility of amide moieties in OmpX/ A8-35 complexes was mapped using the paramagnetic water-soluble relaxation agent $\mathrm{Gd}(\mathrm{DOTA})^{-}$(Fig. 2). In these experiments, the solvent accessibility is quantified by the intermolecular paramagnetic relaxation enhancement effect, as expressed by the relaxivity constant $\varepsilon$ (Caravan 

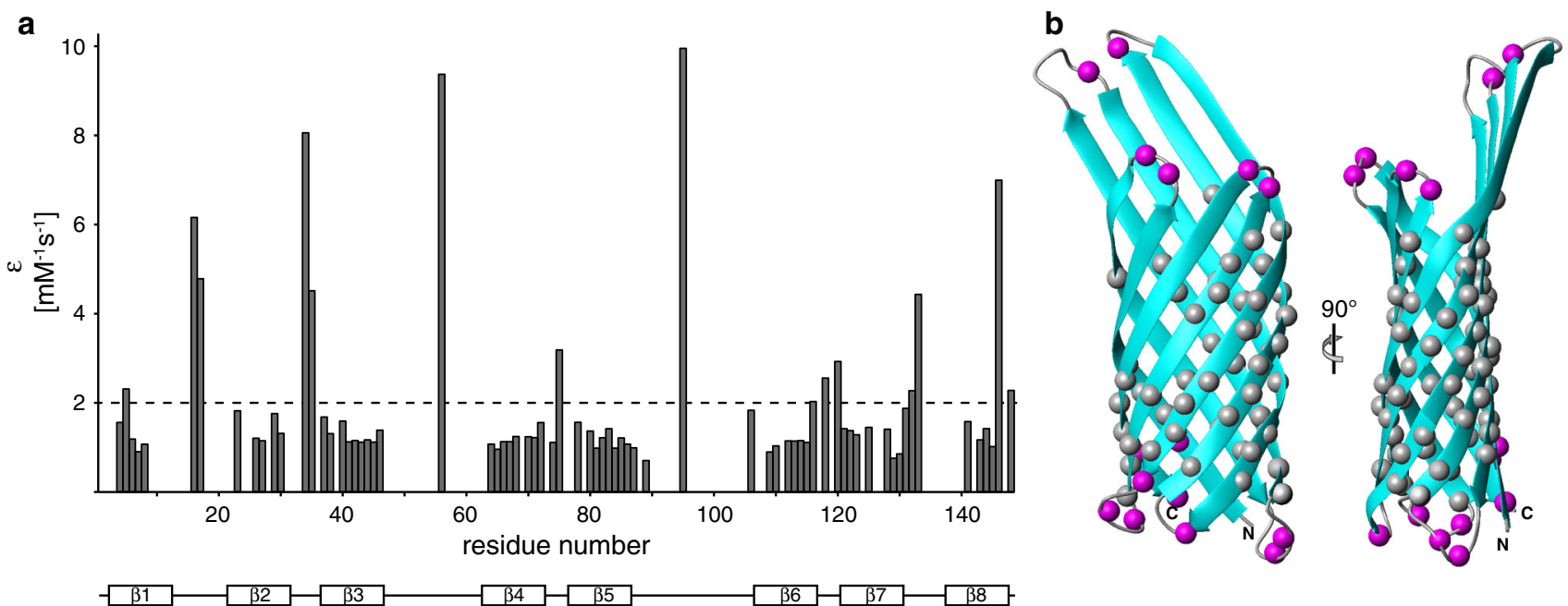

Fig. 2 Surface accessibility of OmpX/A8-35 complexes. a Paramagnetic relaxivity $\varepsilon$ of backbone amide moieties by $\mathrm{Gd}(\mathrm{DOTA})^{-}$, plotted versus the amino acid sequence of OmpX. The secondary structure elements of $\mathrm{OmpX}$ are indicated below. b Structure of OmpX [PDB 1QJ8; Vogt and Schulz (1999)], where all amide

et al. 1999; Hilty et al. 2004). Thereby, $\varepsilon$ is an indicator of the minimal distance that the paramagnet can approach a given amide moiety. Large values of $\varepsilon$ indicate close minimal distances and thus a high solvent accessibility. For amide groups in $\beta$-barrel membrane proteins, differences in $\varepsilon$ correspond to shielding by adsorbed surfactant molecules, either detergent or APol. Using the value of $\varepsilon=2 \mathrm{~s}^{-1} \mathrm{mM}^{-1} \mathrm{mM}$ as threshold level for the classification of amide moieties into a "protected" and an "accessible" group, we find that of the 71 assigned resonances of OmpX in A8-35 amphipols, 57 are protected and 14 are accessible. The accessible amide moieties are located in the turns and loops of OmpX, and the protected amides are located in the barrel region (Fig. 2). In addition, 35 well-resolved, but unassigned resonances were detected in these titration experiments, out of which 30 are accessible and 5 are protected. The narrow chemical shift dispersion of these 35 resonances, suggests that most of them are located in loop and turn regions of OmpX. The relaxivities of OmpX in A8-35 correlate remarkably well with OmpX in the detergent DHPC (Fig. 3). Using the same classification of amide moieties, we find that of the 71 assigned resonances, 54 are protected and 10 are accessible in both conditions (Fig. 3). Only 7 resonances are in different classes in the two preparations. Overall, the solvent accessibility mapping shows that both surfactants adsorb specifically onto the hydrophobic surface of OmpX and in a similar topology (Hilty et al. 2004). These findings are consistent with previous NMR (Catoire et al. 2010) and recent MD (Perlmutter et al. 2014) data. A8-35 thus acts as a suitable membrane mimetic for OmpX. moieties of OmpX in A8-35 that could be unambiguously assigned are shown as spheres. Gray and magenta colors indicate protected and accessible amide moieties, respectively, as classified by the threshold level of $\varepsilon=2 \mathrm{~s}^{-1} \mathrm{mM}^{-1}$ (dashed line in panel a)

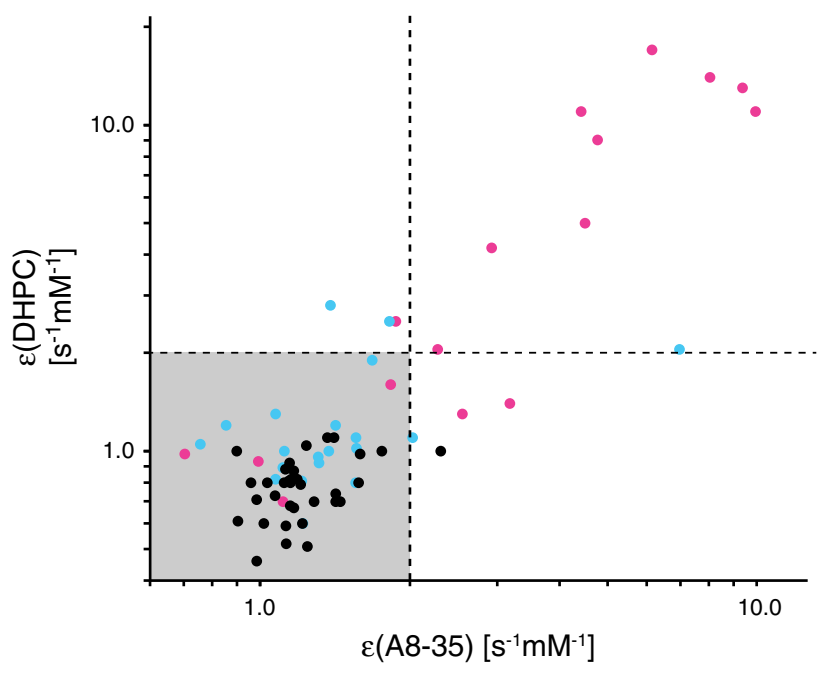

Fig. 3 Comparison of surface accessibilities of OmpX/A8-35 and OmpX/DHPC. Correlations of relaxivities $\varepsilon$ of OmpX/A8-35 determined in the present work for 71 residues are correlated with the corresponding values in OmpX/DHPC (Hilty et al. 2004). Dashed lines denote the threshold level of $\varepsilon<2 \mathrm{~s}^{-1} \mathrm{mM}^{-1}$ used for the classification of residues, and the gray area highlights the residues classified as protected in both conditions. Residues are colored according to their location in the OmpX structure (PDB 1QJ8): black, $\beta$-strand within $>2$ positions from end; magenta, loops and turns; blue, $\beta$-strand within $\leq 2$ positions from end

In a second series of experiments, it was examined how a poly-His tag fused to the N-terminus of the archaebacterial $\alpha$-helical membrane protein BR interacts with each of three different surfactant environments: The detergent DDM, DMPC-based lipid bilayer nanodiscs and A8-35. It has been shown previously that A8-35-trapped BR is both 


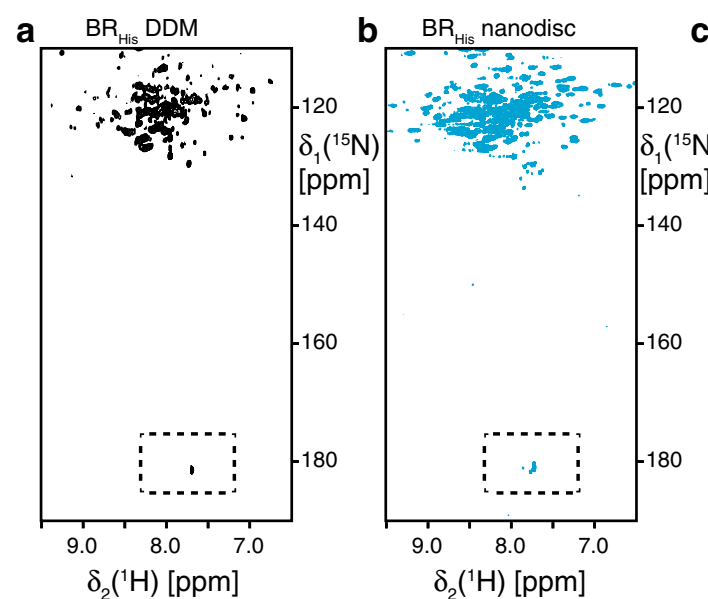

Fig. 4 Differential interaction between an N-terminal poly-His tag on bacteriorhodopsin and selected surfactants. a-c $2 \mathrm{D}\left[{ }^{15} \mathrm{~N},{ }^{1} \mathrm{H}\right]-$ TROSY NMR spectra of BR in a DDM micelles, b DMPC-based lipid bilayer nanodiscs, c amphipol A8-35. d Magnification of the

stable and functional (Dahmane et al. 2013; Gohon et al. 2008), and that its transmembrane, inner core region does not experience significant structural changes when embedded in either of these three different membranemimicking environments (Etzkorn et al. 2013). However, APols and nanodiscs do increase the thermal stability of BR as compared with detergent micelles (Etzkorn et al. 2013; Popot 2010). In addition, NMR data indicate that for several solvent-exposed loop residues, the APol environment shows closest similarity to solid-state NMR results obtained on BR in its native purple membrane (Etzkorn et al. 2013). Here, it was investigated whether a poly-His tag fused at the N-terminus of BR interacts with the surfactant belt. For this assessment, use was made of the differential proton exchange rates of unprotected and protected imidazole protons. Solvent-accessible imidazole protons of the histidine ring feature proton exchange rates with the surrounding water molecules in the fast chemical exchange regime and thus are not detectable in the NMR spectrum (Plesniak et al. 2011). 2D TROSY NMR spectra of BR in DDM solution and in lipid bilayer nanodiscs feature intense correlation crosspeaks in the imidazole spectral region (Fig. 4). These resonances could be unambiguously assigned to the poly-His tag, because BR does not contain any histidine residue in its natural amino acid sequence and because these resonances disappeared in equivalent preparations of the protein after selective cleavage of the poly-His tag with factor Xa (data not shown). The presence of histidine resonances indicates that the tag is at least partially protected from fast exchange with water in DDM and lipid bilayer nanodiscs. Interestingly, the resonances are not detected in BR/A8-35 complexes suggesting that the poly-His tag is directly accessible to the solvent and not embedded in the APol

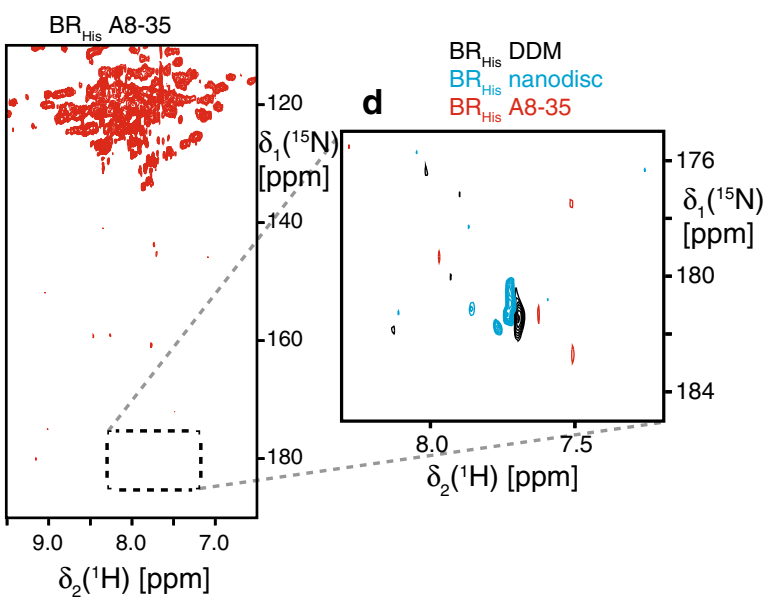

highlighted region, characteristic for aromatic histidine resonance frequencies. Note that BR does not contain any histidine residue in its natural amino acid sequence and thus all of the imidazole $\mathrm{NH}$ resonances arise from the poly-His tag

belt. These observations show a remarkable difference between APols, detergent and nanodiscs, possibly due to the relatively small volume of polar moieties in A8-35 (carboxylate groups), as compared with glycerophosphatidylcholine in nanodiscs and maltoside in DDM.

\section{Conclusion}

As increasingly sophisticated surfactants are developed to handle membrane proteins in aqueous solutions, it becomes apparent that attention should be paid to their effects on membrane protein structure and dynamics, as compared with biological membranes (Zhou and Cross 2013). Recent NMR data, indeed, show that various environments may affect the extramembrane loops of BR (Etzkorn et al. 2013), whereas MD calculations suggest that the dynamics of both the $\beta$-barrel and loops of OmpX are damped in A835 as compared with DHPC (Perlmutter et al. 2014). The present experiments show, using these two membrane proteins as models, that amphipol A8-35 does not detectably interact with either the extramembrane regions of OmpX nor with a tag fused at the N-terminus of BR. Whereas the absence of such contacts cannot be generally inferred, the present data do confirm that APols represent a well-suited medium for biophysical and biochemical studies of integral membrane proteins in aqueous solutions.

Acknowledgments We thank Profs. Kurt Wüthrich and Gerhard Wagner for their kind support of materials and instrument time, Prof. Gerhard Wider for helpful discussions and F. Giusti (UMR 7099) for the synthesis of deuterated A8-35. This work was supported by grants from the German Academic Exchange Service (DAAD) and the DFG (ET 103/2-1) to M.E., as well as the Swiss National Science Foundation (Grant PP00P3_128419) and the European Research Council 
(FP7 contract MOMP 281764) to S.H., by the French Centre National de la Recherche Scientifique, Université Paris-7, and grants from the CNRS interdisciplinary program Physique et Chimie du Vivant, from the EU (BIO4-CT98-0269), from the Human Frontier Science Program Organization (Grant RG00223/2000-M), and from E.U. Specific Targeted Research Project IMPS (Innovative tools for membrane protein structural proteomics) to J.L.P.

\section{References}

Althoff T, Mills DJ, Popot J-L, Kühlbrandt W (2011) Assembly of electron transport chain components in bovine mitochondrial supercomplex $\mathrm{I}_{1} \mathrm{III}_{2} \mathrm{IV}_{1}$. EMBO J 30:4652-4664

Bayburt TH, Sligar SG (2010) Membrane protein assembly into nanodiscs. FEBS Lett 584:1721-1727

Bayburt TH, Grinkova YV, Sligar SG (2002) Self-assembly of discoidal phospholipid bilayer nanoparticles with membrane scaffold proteins. Nano Lett 2:853-856

Caravan P, Ellison JJ, McMurry TJ, Lauffer RB (1999) Gadolinium(III) chelates as MRI contrast agents: structure, dynamics, and applications. Chem Rev 99:2293-2352

Catoire LJ, Zoonens M, van Heijenoort C, Giusti F, Popot J-L, Guittet E (2009) Inter- and intramolecular contacts in a membrane protein/surfactant complex observed by heteronuclear dipole-todipole cross-relaxation. J Magn Res 197:91-95

Catoire LJ, Zoonens M, van Heijenoort C, Giusti F, Guittet E, Popot J-L (2010) Solution NMR mapping of water-accessible residues in the transmembrane $\beta$-barrel of OmpX. Eur Biophys $\mathrm{J}$ 39:623-630

Champeil P, Menguy T, Tribet C, Popot J-L, le Maire M (2000) Interaction of amphipols with the sarcoplasmic reticulum $\mathrm{Ca}^{2+}$. ATPase. J Biol Chem 275:18623-18637

Dahmane T, Rappaport F, Popot J-L (2013) Amphipol-assisted folding of bacteriorhodopsin in the presence and absence of lipids. Functional consequences. Eur Biophys J 42:85-101

Elter, S, Raschle, T, Arens, S, Gelev, V, Etzkorn, M, Wagner, G (2014). The use of amphipols for NMR structural characterization of 7-TM proteins. (submitted to same issue of J. Membr. Biol.)

Etzkorn M, Raschle T, Hagn F, Gelev V, Rice AJ, Walz T, Wagner G (2013) Cell-free expressed bacteriorhodopsin in different soluble membrane mimetics: biophysical properties and NMR accessibility. Structure 21:394-401

Fernández C, Hilty C, Wider G, Guntert P, Wüthrich K (2004) NMR structure of the integral membrane protein OmpX. J Mol Biol 336:1211-1221

Gohon Y, Pavlov G, Timmins P, Tribet C, Popot J-L, Ebel C (2004) Partial specific volume and solvent interactions of amphipol A835. Anal Biochem 334:318-334

Gohon Y, Giusti F, Prata C, Charvolin D, Timmins P, Ebel C, Tribet C, Popot J-L (2006) Well-defined nanoparticles formed by hydrophobic assembly of a short and polydisperse random terpolymer, amphipol A8-35. Langmuir 22:1281-1290

Gohon Y, Dahmane T, Ruigrok R, Schuck P, Charvolin D, Rappaport F, Timmins P, Engelman DM, Tribet C, Popot J-L, Ebel C (2008) Bacteriorhodopsin/amphipol complexes: structural and functional properties. Biophys J 94:3523-3537

Hilty C, Wider G, Fernández C, Wüthrich K (2004) Membrane protein-lipid interactions in mixed micelles studied by NMR spectroscopy with the use of paramagnetic reagents. ChemBioChem 5:467-473
Huynh, KW, Cohen, MR, Moiseenkova-Bell, VY (2014). Application of amphipols for structure-functional analysis of TRP channels. Submitted for publication to the same issue of J. Membr. Biol

Lee D, Hilty C, Wider G, Wüthrich K (2006) Effective rotational correlation times of proteins from NMR relaxation interference. J Magn Reson 178:72-76

Liao M, Cao E, Julius D, Cheng Y (2013) Structure of the TRPV1 ion channel determined by electron cryo-microscopy. Nature 504:107-112

Martinez KL, Gohon Y, Corringer P-J, Tribet C, Mérola F, Changeux J-P, Popot J-L (2002) Allosteric transitions of Torpedo acetylcholine receptor in lipids, detergent and amphipols: molecular interactions $v s$. physical constraints. FEBS Lett 528:251-256

Pautsch A, Vogt J, Model K, Siebold C, Schulz GE (1999) Strategy for membrane protein crystallization exemplified with OmpA and OmpX. Proteins 34:167-172

Perlmutter, JD, Popot, J-L, Sachs, JN (2014). Molecular dynamics simulations of a membrane protein/amphipol complex. (submitted to same issue of J. Membr. Biol.)

Picard M, Dahmane T, Garrigos M, Gauron C, Giusti F, le Maire M, Popot J-L, Champeil P (2006) Protective and inhibitory effects of various types of amphipols on the $\mathrm{Ca}^{2+}$-ATPase from sarcoplasmic reticulum: a comparative study. Biochemistry 45:1861-1869

Planchard, N, Point, E, Dahmane, T, Giusti, F, Renault, M, Le Bon, C, Durand, G, Milon, A, Guittet, E, Zoonens, M, Popot, J-L, Catoire, LJ (2014). The use of amphipols for solution NMR studies of membrane proteins: advantages and limitations as compared to other solubilizing media. (submitted to same issue of J. Membr. Biol.)

Plesniak LA, Mahalakshmi R, Rypien C, Yang Y, Racic J, Marassi FM (2011) Expression, refolding, and initial structural characterization of the $Y$. pestis Ail outer membrane protein in lipids. Biochim Biophys Acta 1808:482-489

Popot J-L (2010) Amphipols, nanodiscs, and fluorinated surfactants: three non-conventional approaches to studying membrane proteins in aqueous solutions. Annu Rev Biochem 79:737-775

Popot J-L, Althoff T, Bagnard D, Banères J-L, Bazzacco P, BillonDenis E, Catoire LJ, Champeil P, Charvolin D, Cocco MJ, Crémel G, Dahmane T, de la Maza LM, Ebel C, Gabel F, Giusti F, Gohon Y, Goormaghtigh E, Guittet E, Kleinschmidt JH, Kühlbrandt W, Le Bon C, Martinez KL, Picard M, Pucci B, Rappaport F, Sachs JN, Tribet C, van Heijenoort C, Wien F, Zito F, Zoonens M (2011) Amphipols from A to Z. Annu Rev Biophys 40:379-408

Raschle T, Hiller S, Etzkorn M, Wagner G (2010) Nonmicellar systems for solution NMR spectroscopy of membrane proteins. Curr Opin Struct Biol 20:471-479

Tribet C, Audebert R, Popot J-L (1996) Amphipols: polymers that keep membrane proteins soluble in aqueous solutions. Proc Natl Acad Sci USA 93:15047-15050

Vogt J, Schulz GE (1999) The structure of the outer membrane protein OmpX from Escherichia coli reveals possible mechanisms of virulence. Structure 7:1301-1309

Zhou HX, Cross TA (2013) Influences of membrane mimetic environments on membrane protein structures. Annu Rev Biophys 42:361-392

Zoonens M, Popot J-L (2014). Amphipols for each season. (to be submitted to same issue of J. Membr. Biol.)

Zoonens M, Catoire LJ, Giusti F, Popot J-L (2005) NMR study of a membrane protein in detergent-free aqueous solution. Proc Natl Acad Sci USA 102:8893-8898 